\title{
Gemeinsamer nationaler Bericht des BVL und RKI zu lebensmittelbedingten Krankheitsausbrüchen in Deutschland, 2015
}

\author{
Bettina Rosner ${ }^{1} \cdot$ Thomas Schewe $^{2}$
}

Published online: 17 December 2016

(c) The Author(s) 2016. Dieser Artikel ist auf Springerlink.com mit Open Access verfügbar.

Zusammenfassung In Deutschland werden jedes Jahr Hunderte von Krankheitsausbrüchen registriert, die über mikrobiell kontaminierte Lebensmittel verursacht wurden. Die Daten zu lebensmittelbedingten Ausbrüchen werden von den Gesundheits- und Lebensmittelüberwachungsbehörden vor Ort erfasst und über zwei parallele Meldewege (Übermittlung nach dem Infektionsschutzgesetz bzw. BELA-Meldungen) über die Behörden der Länder an das Robert Koch-Institut (RKI) bzw. an das Bundesamt für Verbraucherschutz und Lebensmittelsicherheit (BVL) übermittelt. Die Daten werden vom RKI und vom BVL gemeinsam bewertet und an die Europäische Behörde für Lebensmittelsicherheit (European Food Safety Authority, EFSA) berichtet. Erstmals werden die Daten zu lebensmittelbedingten Krankheitsausbrüchen in Deutschland jetzt auch in Form eines nationalen Berichts vom BVL und RKI gemeinsam veröffentlicht. Im Jahr 2015 wurden insgesamt 384 lebensmittelbedingte Krankheitsausbrüche an das RKI bzw. an das BVL übermittelt. Mindestens 2.072 Erkrankungen und 224 Hospitalisierungen standen mit den Ausbrüchen in Zusammenhang. Todesfälle sind nicht aufgetreten. Bei 28 Krankheitsausbrüchen wurde die Evidenz des

Thomas Schewe

108@bvl.bund.de

1 Robert Koch-Institut (RKI), Seestraße 10, 13353 Berlin, Germany

2 Bundesamt für Verbraucherschutz und Lebensmittelsicherheit (BVL), Mauerstr. 39-42, 10117 Berlin, Germany
Zusammenhangs zwischen den Erkrankungen und einem ursächlichen Lebensmittel gemäß EFSA-Kriterien als hoch eingestuft. Den größten Anteil davon (12/28, 43\%) nahmen Ausbrüche durch den Erreger Campylobacter ein. Bei allen 12 Campylobacter-Ausbrüchen mit hoher Evidenz war nicht abgekochte Rohmilch das verursachende Lebensmittel. Weitere Erreger und Agenzien, die Ausbrüche mit hoher Evidenz verursachten, waren Histamin (z.B. in Thunfisch) (5/28, 18\%), Salmonella spp. (3/28, 11\%), Bacillus cereus (2/28, 7\%), Staphylococcus aureus, Norovirus, Listeria monocytogenes, Trichinella, Clostridium perfringens und Ciguatoxin (jeweils 1 Ausbruch). Der Ausbruch mit den meisten Erkrankungsfällen ( $\mathrm{n}=159$ ), davon 149 Kinder, wurde durch Listeria monocytogenes verursacht. Die Betroffenen dieses Gastroenteritis-Ausbruchs hatten in Einrichtungen zur Kindertagesbetreuung Milchreis von einem Caterer gegessen, in dem L. monocytogenes in sehr hoher Konzentration nachgewiesen werden konnte. Es ist davon auszugehen, dass jährlich deutlich mehr lebensmittelbedingte Ausbrüche in Deutschland auftreten, die 384 gemeldeten und an das BVL bzw. das RKI übermittelten Ausbrüche also nur die Spitze des Eisbergs darstellten. Gemeinsame, interdisziplinäre Ausbruchsuntersuchungen durch die zuständigen Gesundheitsämter und Lebensmittelüberwachungsbehörden sind unerlässlich, um die Ausbruchsursachen zu erkennen und geeignete Maßnahmen ergreifen sowie Empfehlungen aussprechen $\mathrm{zu}$ können, die ein Ausbruchsgeschehen beenden und weitere Ausbrüche verhindern können. 


\section{Einleitung}

Jedes Jahr erkranken in Deutschland zahlreiche Menschen mit akuten gastrointestinalen Symptomen durch eine lebensmittelbedingte Infektion mit bakteriellen, viralen oder parasitären Erregern oder durch Lebensmittelvergiftungen, die meist von bakteriellen Toxinen ausgelöst werden. Viele potenziell lebensmittelbedingte Krankheiten, z.B. Salmonellosen oder Campylobacter-Enteritiden, sind gemäß Infektionsschutzgesetz (IfSG) meldepflichtig. Ermittlungen zur Art, Ursache, Ansteckungsquelle und Ausbreitung der Erkrankungen gehören zu den Aufgaben des Gesundheitsamtes (§25 IfSG). Daten zu den Erkrankungen werden anonymisiert von den Gesundheitsämtern über die Landesgesundheitsbehörden an das Robert Koch-Institut (RKI) übermittelt (\$11 IfSG). Wird bei zwei oder mehr akuten infektiösen Gastroenteritis-Erkrankungen oder einer mikrobiell bedingten Lebensmittelvergiftung ein epidemischer Zusammenhang vermutet oder ist ein solcher Zusammenhang wahrscheinlich, werden diese Erkrankungen als Fälle eines Krankheitsausbruchs zusammengeführt. Als lebensmittelbedingt oder zumindest als wahrscheinlich lebensmittelbedingt wird ein Krankheitsausbruch bewertet, wenn die Erkrankungen in Zusammenhang mit einem Lebensmittel stehen, das verdächtigt wird, mit einem Erreger oder durch ein Toxin kontaminiert gewesen zu sein, oder das labordiagnostisch nachweislich kontaminiert war. Daten zu Erkrankungen durch lebensmittelbedingte Krankheitsausbrüche, die gemäß IfSG an das RKI übermittelt wurden, werden im Infektionsepidemiologischen Jahrbuch vom RKI veröffentlicht (Robert Koch-Institut 2016).

Parallel zu den Untersuchungen der Gesundheitsämter sind die zuständigen Lebensmittelüberwachungsbehörden (LMÜ) der Landkreise und kreisfreien Städte verpflichtet, Ermittlungen und ggf. Maßnahmen einzuleiten, wenn der Verdacht besteht, dass ein in Verkehr gebrachtes Lebensmittel gesundheitsschädlich sein könnte. Dies gilt insbesondere, wenn bereits Erkrankungen aufgetreten sind und sich das verdächtige Lebensmittel noch auf dem Markt befinden könnte. Die LMÜ werden über einen möglichen lebensmittelbedingen Krankheitsausbruch durch die zuständigen Gesundheitsämter informiert oder erhalten Beschwerden von erkrankten Verbrauchern.

Ein zeitnaher Informationsaustausch zwischen Gesundheits- und Lebensmittelüberwachungsbehörden ist vom Gesetzgeber ausdrücklich gewünscht. Im Nachgang zu dem großen lebensmittelbedingten EHEC-Ausbruch in Deutschland im Jahr 2011 wurden sowohl im Infektionsschutzgesetz (§27 Abs. 1 IfSG) als auch im Lebensmittel- und Futtermittelgesetzbuch ( $\$ 42$ Abs. 3 LFGB) Ergänzungen vorgenommen, die den Informationsaustausch zwischen den Behörden bei einem lebensmittelbedingten Krankheitsausbruch oder dem Verdacht darauf festlegen. Ziel der Zusammenarbeit zwischen Gesundheits- und Lebensmittelüberwachungsbehörden bei der Aufklärung von lebensmittelbedingten Krankheitsausbrüchen ist es, möglichst schnell die Infektionsquelle für die aufgetretenen Erkrankungen $\mathrm{zu}$ ermitteln und weitere Gesundheitsschäden für die Verbraucher zu vermeiden. Dabei können die Befragungen von Erkrankten und ggf. analytische epidemiologische Studien der Gesundheitsbehörden entscheidende Erkenntnisse für die Ermittlung der Quelle der Krankheitshäufungen liefern.

Die Lebensmittelüberwachungsbehörden können ergänzend durch Betriebskontrollen und Lebensmittelproben kontaminierte Lebensmittel und Hygieneprobleme in Lebensmittelbetrieben identifizieren und die Vertriebswege von Produkten ermitteln. Sie sind auf der Grundlage des Lebensmittelrechts auch dazu bevollmächtigt, ggf. Auflagen gegenüber Lebensmittelbetrieben zu erlassen oder Verkehrsverbote für bestimmte Lebensmittel $\mathrm{zu}$ verhängen.

Um möglichst viele Informationen über die Umstände, die zu lebensmittelbedingten Ausbrüchen geführt haben, zu sammeln und über die daraus gewonnenen Erkenntnisse zukünftige lebensmittelbedingte Ausbrüche vermeiden zu können, werden in Deutschland und anderen europäischen Ländern neben den Daten zu den Erkrankungen durch lebensmittelbedingte Ausbrüche auch Daten zu den am Ausbruch beteiligten Lebensmitteln, einschließlich deren Herstellung und Bearbeitung, erfasst. In Deutschland erfolgt dies über ein bundeseinheitliches System zur Erfassung von Daten zu Lebensmitteln, die an Krankheitsausbrüchen beteiligt sind (Akronym BELA). Informationen $\mathrm{zu}$ den Lebensmitteln werden von den lokalen LMÜ erhoben und als BELA-Meldung über die zuständigen Behörden der Länder an das Bundesamt für Verbraucherschutz und Lebensmittelsicherheit (BVL) übermittelt. In den Jahren bis 2015 wurden die BELADaten vom Bundesinstitut für Risikobewertung (BfR) erfasst, ausgewertet und veröffentlicht (Bundesinstitut für Risikobewertung 2015a). Diese Aufgabe wurde 
im Jahr 2015 vom BVL übernommen. Die Datenerfassung erfolgt gemäß $\S 11$ der bundesweit einheitlichen Allgemeinen Verwaltungsvorschrift (AVV) über die Erfassung, Auswertung und Veröffentlichung von Daten über das Auftreten von Zoonosen und Zoonoseerregern entlang der Lebensmittelkette (AVV Zoonosen Lebensmittelkette 2012), durch die in Deutschland die Richtlinie 2003/99/EG der Europäischen Union zur Überwachung von Zoonosen und Zoonoseerregern umgesetzt wird (Richtlinie 2003/99/EG des Europäischen Parlaments und des Rates). Diese beinhaltet, dass Daten zu lebensmittelbedingten Krankheitsausbrüchen von den EU-Mitgliedsländern jährlich an die Europäische Behörde für Lebensmittelsicherheit (European Food Safety Authority, EFSA) berichtet werden müssen.

Der Bericht an die EFSA wird vom BVL gemeinsam mit dem RKI erstellt. Grundlage sind die BELA-Daten und die gemäß IfSG an das RKI übermittelten Daten zu lebensmittelbedingten Krankheitsausbrüchen. Erstmals werden diese Daten jetzt auch in Form eines nationalen Berichts $\mathrm{zu}$ lebensmittelbedingten Krankheitsausbrüchen in Deutschland vom BVL und RKI gemeinsam veröffentlicht.

\subsection{Abgleich der BELA- und IfSG-Meldedaten zu lebensmittelbedingten Krankheitsausbrüchen in Deutschland}

Grundlage für den Bericht an die EFSA bzw. den nationalen Bericht ist ein Datenabgleich der BELAund IfSG-Meldedaten zu lebensmittelbedingten Ausbrüchen zwischen BVL und RKI. Theoretisch wäre zu erwarten, dass für jeden lebensmittelbedingten Ausbruch, der dem Gesundheitsamt bekannt wird, nicht nur eine Übermittlung der Daten gemäß IfSG an das RKI erfolgt, sondern von der zuständigen LMÜ auch ein BELA-Bogen ausgefüllt und an das BVL übermittelt wird. Umgekehrt wäre zu erwarten, dass jeder lebensmittelbedingte Ausbruch, der der LMÜ bekannt wird und für den ein BELA-Bogen vorliegt, auch über das zuständige Gesundheitsamt im IfSGMeldesystem übermittelt wird. In der Praxis ist dies aber häufig nicht der Fall. Die Anzahl der über das IfSG-Meldesystem jährlich an das RKI übermittelten lebensmittelbedingten Ausbrüche übersteigt die Anzahl der BELA-Meldungen etwa um das Fünffache. Außerdem werden über BELA Ausbrüche übermittelt, die nicht als lebensmittelbedingte Ausbrüche im IfSG-Meldesystem auftauchen. Mehrmals im Jahr tauschen BVL und RKI die vorliegenden Daten zu lebensmittelbedingten Ausbrüchen aus. Bei Ausbrüchen, die im IfSG-Meldesystem mit 5 oder mehr
Fällen übermittelt wurden oder bei denen angegeben ist, dass ein Erregernachweis im Lebensmittel erfolgte, eine Beteiligung der LMÜ also impliziert ist, und für die (noch) keine BELA-Bögen vorliegen, werden vom BVL über die Lebensmittelüberwachungsbehörden der Länder Informationen zum Ausbruch eingeholt und die entsprechenden BELABögen erbeten. Bei Ausbrüchen mit BELA-Bogen, die nicht über das IfSG-Meldesystem übermittelt wurden, erbittet das RKI die für den EFSA-Bericht erforderlichen Informationen (Anzahl der Erkrankungen, Hospitalisierungen, Todesfälle) über die Landesgesundheitsbehörden von den Gesundheitsämtern, regt eine Übermittlung des Krankheitsausbruchs an oder bittet ggf. um Änderung der Kategorie des Ausbruchs in den Meldedaten in „lebensmittelbedingt“. Rechtzeitig vor dem Datenschluss für den EFSA-Bericht (31.5. des Folgejahres) werden die vorliegenden Daten von BVL und RKI zusammengestellt und nach den aktuellen EFSA-Kriterien bewertet.

\subsection{Berichterstattung an die EFSA}

Seit dem Jahr 2007 werden lebensmittelbedingte Ausbrüche jährlich über ein harmonisiertes Berichterstattungssystem an die EFSA berichtet. Seit 2010 erfolgt die Berichterstattung an die EFSA gemäß dem European Union Food-borne Outbreak Reporting System (EU-FORS).

$\mathrm{Zu}$ den Pflichtangaben gehören die Gesamtzahl der lebensmittelbedingten Ausbrüche, die Anzahl der damit in Zusammenhang stehenden Erkrankungen, Hospitalisierungen und Todesfälle, der den Ausbruch verursachenden Erreger, das/die verdächtigte/n Lebensmittel, die Kategorie des Ortes, wo das implizierte Lebensmittel produziert/verarbeitet/verkauft/ verzehrt wurde (z.B. Restaurant/Kantine) und Faktoren, die zum Ausbruch beigetragen haben könnten (z.B. nicht ausreichende Erhitzung des Lebensmittels). Im EU-FORS-System wird für jeden berichteten Ausbruch ein Evidenzgrad für den Zusammenhang zwischen den Erkrankungen und einem verdächtigten Lebensmittel festgelegt. Dabei wird zwischen lebensmittelbedingten Ausbrüchen mit hoher (,strong evidence“) und niedriger Evidenz (,weak evidence“) unterschieden (European Food Safety Authority 2011). In den Jahren bis 2010 waren Ausbrüche als "bestätigt" (verified) oder „möglicherweise“ (possible) lebensmittelassoziiert definiert und als solche an die EFSA übermittelt worden. Ausbrüche mit hoher Evidenz werden einzeln berichtet, Ausbrüche mit niedriger Evidenz können aggregiert an die EFSA berichtet werden. Die 
Kriterien, mit denen die Evidenz zu bewerten ist, werden von der EFSA definiert. In Deutschland bewerten BVL und RKI den Evidenzgrad anhand der vorliegenden Daten gemeinsam. Nach der aktuellen EFSA-Definition (European Food Safety Authority 2016) wird zwischen epidemiologischer Evidenz, mikrobiologischer Evidenz, Evidenz aus Umgebungsuntersuchungen („,descriptive environmental evidence“) und Evidenz aus Produktverfolgungen (,product-tracing investigations“) unterschieden. Der Grad der Evidenz (hoch oder niedrig) ist unabhängig von der Art der Evidenz. Die epidemiologische Evidenz ist als hoch zu bewerten, wenn in einer gut durchgeführten analytischen epidemiologischen Studie (Kohortenstudie oder Fall-Kontroll-Studie) ein statistisch signifikanter Zusammenhang zwischen einem Lebensmittel und den Erkrankungen ermittelt wurde oder wenn die vorliegende deskriptive epidemiologische Evidenz überzeugend ist. Hohe mikrobiologische Evidenz liegt vor, wenn der die Erkrankungen verursachende Erreger im Lebensmittel oder in seinen Zutaten, in der Lebensmittelkette oder in der Umgebung des Herstellungs- oder Verarbeitungsprozesses nachgewiesen werden konnte. Konnte bei den Erkrankten kein Erreger nachgewiesen werden, kann die mikrobiologische Evidenz nur hoch sein, wenn die Symptome der Erkrankten für diesen Erreger typisch (pathognomonisch) waren. Evidenz aus Produktverfolgungen schließt alle Stufen der Lebensmittelkette ein (Produktion, Verarbeitung und Vertrieb). Bei Rückwärtsverfolgungen („,traceback“) wird das Lebensmittelprodukt (oder seine Zutaten) vom Verkaufsort (point of sale) zu seiner Quelle (bestenfalls der landwirtschaftliche Betrieb) zurückverfolgt, während bei Vorwärtsverfolgungen („,trace-forward“) der Weg des Lebensmittelprodukts von der Quelle (landwirtschaftlicher Betrieb) zum Verkaufsort nachvollzogen wird.

Nach dem finalen Datenabgleich zwischen BVL und RKI werden die Ausbrüche, die über das BELASystem, das IfSG-Meldesystem oder über beide Systeme übermittelt wurden, als solche mit hoher und solche mit niedriger Evidenz bewertet. Dabei werden zunächst die Ausbrüche mit hoher Evidenz gemäß EFSA-Definition identifiziert. Die Gesamtzahl der Ausbrüche mit niedriger Evidenz entspricht der Summe der lebensmittelbedingten Ausbrüche mit niedriger Evidenz, die über das IfSG-Meldesystem übermittelt wurden, und der Ausbrüche mit niedriger Evidenz, die ausschließlich über das BELA-System übermittelt wurden, weil der Nachweis des Erregers/des Agens bei Erkrankten nicht nach IfSG meldebzw. übermittlungspflichtig ist (z.B. Ausbrüche durch
Histamin). Ausbrüche mit hoher Evidenz werden einzeln mit detaillierten Angaben (Erreger, Lebensmittel, Evidenz, Setting des Ausbruchs, Herkunft des Lebensmittels, Umstände, die zur Kontamination des Lebensmittels geführt haben könnten, sowie Anzahl der Erkrankungen, Hospitalisierungen und Todesfälle) an die EFSA übermittelt. Ausbrüche mit niedriger Evidenz werden nach Erregerkategorien aggregiert und nur mit Angaben zur Anzahl der Erkrankungen, Hospitalisierungen und Todesfällen übermittelt. Es besteht die Möglichkeit, auch Ausbrüche, bei dem der Erreger unbekannt blieb (Erregerkategorie „Unknown“), und Ausbrüche, bei denen die Anzahl der Erkrankungen nicht bekannt ist (Angabe „Unknown“ bzw. -1), zu übermitteln.

\section{Ergebnisse}

Im Folgenden werden die Daten für das Jahr 2015, die gemäß IfSG an das RKI bzw. die gemäß BELA an das BVL übermittelt wurden, kurz einzeln dargestellt. Im Anschluss folgt die ausführlichere Darstellung der Daten, die nach dem gemeinsamen Datenabgleich zwischen den Behörden an die EFSA übermittelt wurden.

\subsection{Gemäß IfSG an das RKI übermittelte lebensmittelbedingte Krankheitsausbrüche im Jahr 2015}

Im Jahr 2015 wurden an das RKI insgesamt 337 lebensmittelbedingte Ausbrüche übermittelt, davon 19 lebensmittelbedingte Norovirus-Ausbrüche und 318 Ausbrüche durch andere Erreger (Robert KochInstitut 2016). Die Norovirus-Ausbrüche werden bei der Auswertung der IfSG-Meldedaten gesondert betrachtet, da wegen Besonderheiten bei der Falldefinition die Vergleichbarkeit mit anderen Erkrankungszahlen nicht gegeben ist. Seit 2011 erfüllt nur noch die Kategorie der klinisch-labordiagnostisch bestätigten Norovirus-Fälle die Referenzdefinition, die Grundlage der Daten ist, die vom RKI veröffentlicht werden. Norovirus-Ausbrüche enthalten häufig klinisch-epidemiologisch bestätigte Erkrankungen ohne labordiagnostischen Erregernachweis. Diese erfüllen die Referenzdefinition nicht. Im Infektionsepidemiologischen Jahrbuch des RKI werden nur Norovirus-Ausbrüche berichtet, die mindestens 2 klinisch-labordiagnostisch bestätigte Erkrankungen enthalten. Dadurch kommt es zu einer deutlichen Unterschätzung der tatsächlichen Anzahl der Norovirus-Ausbrüche sowie der im Rahmen von 
Norovirus-Ausbrüchen übermittelten Fallzahlen (Robert Koch-Institut 2016).

Die häufigsten gemäß IfSG übermittelten lebensmittelbedingten Krankheitsausbrüche wurden durch Campylobacter spp. (179/318, 56\%) verursacht, am zweithäufigsten waren Ausbrüche durch Salmonella spp. (106/318, 33\%). Andere Erreger (ohne Norovirus) verursachten die restlichen $11 \%$ der übermittelten Krankheitsausbrüche. In den letzten Jahren hat die Zahl der als lebensmittelbedingt übermittelten Ausbrüche (ohne Norovirus-Ausbrüche) insgesamt um etwa 18\% von 390 Ausbrüchen im Jahr 2010 auf 318 Ausbrüche im Jahr 2015 abgenommen. Dies lässt sich im Wesentlichen auf den Rückgang der Salmonellosen und, damit einhergehend, auf die Abnahme der Salmonellose-Ausbrüche um etwa die Hälfte von 214 Ausbrüchen im Jahr 2010 auf 106 Ausbrüche im Jahr 2015 zurückführen. Die Zahl der übermittelten Campylobacter-Enteritis-Ausbrüche ist im gleichen Zeitraum von 149 (2010) auf 179 gestiegen (Zunahme um 20\%). Der relative Anteil der Campylobacter-Enteritis-Ausbrüche an den übermittelten lebensmittelbedingten Ausbrüchen ist von 38\% (2010) auf 56\% (2015) angestiegen, während der Anteil der SalmonelloseAusbrüche von 55\% (2010) auf 33\% (2015) abnahm. Die Mehrzahl (88\%) der gemäß IfSG übermittelten lebensmittelbedingten Ausbrüche (ohne Norovirus) betraf nur jeweils weniger als 5 Fälle. Größere Ausbrüche (5 oder mehr Fälle) wurden durch Salmonella spp. (23/106, $22 \%)$ und Campylobacter spp. (13/179, 7\%) verursacht. Es wurden auch ein größerer Listeriose-Ausbruch und ein Trichinellose-Ausbruch übermittelt.

\section{2 Über BELA an das BVL gemeldete lebensmittelbedingte Krankheitsausbrüche im Jahr 2015}

Im Jahr 2015 wurden 69 BELA-Meldungen an das BVL gesendet. Von diesen lag bei 54 ein lebensmittelbedingter Krankheitsausbruch gemäß der Definition der Richtlinie 2003/99/EG (Richtlinie 2003/99/EG des Europäischen Parlaments und des Rates) vor. Die übrigen Meldungen wurden nicht als lebensmittelbedingter Ausbruch bewertet, weil nur ein Erkrankungsfall bekannt war, also keine Erkrankungshäufung erkennbar war, nach Abschluss der Ermittlungen kein Bezug zu einem Lebensmittel hergestellt werden konnte oder die wahrscheinlichere Ursache des Ausbruchs die Übertragung des Erregers von Mensch zu Mensch war. Gegenüber dem Vorjahr (2014: 48 BELA-Meldungen) hat die Anzahl der Meldungen um 13\% zugenommen. Von 2010 bis 2014 war eine kontinuierliche Abnahme der BELA-
Meldungen von 94 (im Jahr 2010) auf 48 (2014) beobachtet worden.

Bei den ursächlichen Erregern bzw. Toxinen war Campylobacter spp. am häufigsten vertreten (15/54, $28 \%)$, gefolgt von Salmonella spp. $(13 / 54,24 \%)$ und Histamin (5/54, 9\%).

\subsection{An die EFSA berichtete lebensmittelbedingte Ausbrüche mit hoher Evidenz im Jahr 2015}

Die kombinierten Daten aus den Übermittlungen an RKI und BVL ergaben für Deutschland im Jahr 2015 insgesamt 384 lebensmittelbedingte Krankheitsausbrüche. Mindestens 2.072 Erkrankungen und 224 Hospitalisierungen standen mit diesen Ausbrüchen in Zusammenhang. Bei drei Ausbrüchen blieb die Zahl der Erkrankungen/Hospitalisierungen unbekannt. Todesfälle wurden nicht verursacht.

Insgesamt 28 Ausbrüche (7\%) wurden als Ausbrüche mit hoher Evidenz eingestuft. Hier war eine Verbindung zwischen den Erkrankungsfällen und einem ursächlichen Lebensmittel hinreichend wahrscheinlich. Die Anzahl der Ausbrüche mit hoher Evidenz war genauso hoch wie im Vorjahr (2014: 28). Der hohe Evidenzgrad ergab sich bei 19 Ausbrüchen (68\%) über den Nachweis des Erregers/Agens im Lebensmittel oder in seinen Zutaten. Bei einem Ausbruch erfolgte der Erreger-/Agensnachweis in Proben entlang der Lebensmittelkette oder aus der Umgebung des Herstellungs- oder Verarbeitungsprozesses. Bei 7 Ausbrüchen (25\%) wurde die deskriptive epidemiologische Evidenz als hinreichend überzeugend eingeschätzt, ohne dass ein Erreger im Lebensmittel nachgewiesen werden konnte. Bei einem Ausbruch wurde der Zusammenhang zwischen Erkrankungen und einem verdächtigten Lebensmittel über eine analytische epidemiologische Studie hergestellt. Ausbrüche mit hoher Evidenz führten zu mindestens 619 Erkrankungen (30\% aller Erkrankungen durch lebensmittelbedingte Ausbrüche) und 53 Hospitalisierungen (24\% aller Hospitalisierungen). Bei einem Ausbruch, der durch Histamin verursacht wurde, blieb die Zahl der Erkrankungen unbekannt.

Ausbrüche durch den Erreger Campylobacter spp. nahmen den größten Anteil unter den Ausbrüchen mit hoher Evidenz ein (12/28, 43\%). Gegenüber dem Vorjahr konnte eine deutliche Zunahme beobachtet werden (2014: 4/28, 14\%). Der Anteil der Campylobacter-Ausbrüche unter den Ausbrüchen mit hoher Evidenz war im Jahr 2015 erstmals höher als der Anteil der Ausbrüche durch Salmonella spp. (2014: 9/28, 32\%). Der hohe Evidenzgrad wurde bei 5 Ausbrüchen allein durch die überzeugende deskriptive 
epidemiologische Evidenz erreicht, bei den übrigen 7 Ausbrüchen konnte zusätzlich der Erreger im Lebensmittel (Rohmilch) labordiagnostisch nachgewiesen werden. Bei 11 der 12 CampylobacterAusbrüche wurde als Erreger Campylobacter jejuni identifiziert, bei einem Ausbruch wurde die Campylobacter-Spezies nicht bestimmt oder nicht angegeben. Weitere Erreger und Agenzien, die Ausbrüche mit hoher Evidenz verursachten, waren Histamin $(5 / 28,18 \%)$, Salmonella spp. $(3 / 28,11 \%)$, Bacillus cereus $(2 / 28,7 \%)$, Staphylococcus aureus, Norovirus, Listeria monocytogenes, Trichinella, Clostridium perfringens und Ciguatoxin (jeweils 1 Ausbruch) (Tab. 1).

Bei 12 Ausbrüchen mit hoher Evidenz (12/28, 43\%) konnte Milch, und zwar explizit nicht abgekochte Rohmilch, als auslösendes Lebensmittel identifiziert werden. Bei allen 12 Ausbrüchen war Campylobacter der ursächliche Erreger. Lebensmittelkategorien, die mit den Ausbrüchen mit hoher Evidenz am zweithäufigsten in Zusammenhang standen, waren Fisch und Fischerzeugnisse (5/28, 18\%) und zusammengesetzte Lebensmittel (EFSA-Kategorie „mixed

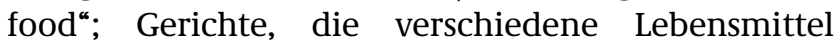
umfassen, z.B. Kartoffelsalat) (5/28, 18\%). Andere genannte Lebensmittelkategorien waren Gemüse und Gemüseerzeugnisse (2), Früchte, Beeren und Erzeugnisse daraus (1), Backwaren (1) und Fleisch und Fleischerzugnisse (1). Gegenüber dem Vorjahr hat der Anteil der Ausbrüche, die durch Fleisch und Fleischerzeugnisse verursacht wurden, abgenommen (2014: 6/28, 21\%), während der Anteil der Ausbrüche durch
Milch zugenommen hat (2014: 2/28, 7\%). Campylobacter und Rohmilch stellte die am häufigsten gemeldete Kombination aus Erreger/Agens und ursächlichem Lebensmittel dar. An zweiter Stelle folgten Krankheitsausbrüche durch Histamin in Thunfisch $(4 / 28,14 \%)$.

Als Ursprungsort der Kontamination bzw. des Hygienemangels wurde bei 7 Ausbrüchen mit starker Evidenz (25\%) die Kategorie „Haushalt“ angegeben. Bei 6 Ausbrüchen (21\%) wurde die Kategorie „Restaurant/Café/Pub/Bar/Hotel/Catering“ genannt. Die Kategorie „Landwirtschaftlicher Betrieb“ wurde in 4 Ausbrüchen (14\%) als Ursprungsort des Problems angegeben. Jeweils einmal wurden die Kategorien „Schule/Kindergarten“ und „Anderer Ort“ genannt. Bei 9 Ausbrüchen war es nicht möglich, den Ursprungsort des Problems zu identifizieren.

Der Ausbruch mit den meisten Erkrankungsfällen ( $\mathrm{n}=159$ ), davon 149 Kinder, wurde durch Listeria monocytogenes verursacht. Die Betroffenen dieses Gastroenteritis-Ausbruchs hatten in Einrichtungen zur Kindertagesbetreuung Milchreis von einem Caterer gegessen, in dem L. monocytogenes in sehr hoher Konzentration nachgewiesen werden konnte (Robert Koch-Institut 2016). Wahrscheinlich führte ein mangelhafter Abkühlungsprozess beim Herstellerbetrieb zur Vermehrung des Erregers im Lebensmittel.

Ein weiterer großer Ausbruch mit 90 Erkrankungsfällen ereignete sich in Einrichtungen zur Gemeinschaftsverpflegung und betraf auch Personen, die mit Essen auf Rädern versorgt wurden. In

Tab. 1 An die EFSA übermittelte lebensmittelbedingte Ausbrüche mit hoher Evidenz, nach Erregern, Deutschland 2015 ( $n=28$ )

\begin{tabular}{|c|c|c|c|c|c|}
\hline Erreger/Agens & $\begin{array}{l}\text { Anzahl } \\
\text { Ausbrüche }\end{array}$ & $\begin{array}{l}\text { Anteil } \\
(\%)\end{array}$ & Anzahl Fälle & $\begin{array}{l}\text { Anzahl } \\
\text { Hospitalisierungen }\end{array}$ & $\begin{array}{l}\text { Anzahl } \\
\text { Todesfälle }\end{array}$ \\
\hline Campylobacter spp. & 12 & 42,9 & 153 & 13 & 0 \\
\hline Histamin & 5 & 17,9 & Unbekannt (mind. 21) & Unbekannt & 0 \\
\hline Salmonella Enteritidis & 2 & 7,1 & 60 & 27 & 0 \\
\hline Salmonella Typhimurium & 1 & 3,6 & 17 & 7 & 0 \\
\hline Bacillus cereus & 2 & 7,1 & 94 & Unbekannt & 0 \\
\hline Listeria monocytogenes & 1 & 3,6 & 159 & 2 & 0 \\
\hline Norovirus & 1 & 3,6 & 73 & 1 & 0 \\
\hline Staphylococcus aureus/ Staphlyokokken Enterotoxin & 1 & 3,6 & 25 & 0 & 0 \\
\hline Trichinella & 1 & 3,6 & 8 & 3 & 0 \\
\hline Marines Biotoxin (Ciguatoxin) & 1 & 3,6 & 6 & 0 & 0 \\
\hline Clostridium perfringens & 1 & 3,6 & 3 & 0 & 0 \\
\hline GESAMT & 28 & $100^{\mathrm{a}}$ & 619 (mind.) $^{\mathrm{b}}$ & 53 (mind.) $^{b}$ & 0 \\
\hline
\end{tabular}

a Die berechneten Prozentzahlen wurden gerundet. Deshalb ergibt die Summe nicht genau 100,0\%

b Bei einigen Ausbrüchen konnte die Fallzahl bzw. die Anzahl der Hospitalisierungen nicht oder nicht genau ermittelt werden. Die Summenangabe stellt deshalb eine Mindestanzahl dar 
Rückstellproben mehrerer Menükomponenten wurde Bacillus cereus nachgewiesen, darunter Schweinegeschnetzeltes, Kartoffeln und Nudeln. Die Rückstellproben waren unmittelbar nach der Zubereitung und vor der weiteren Portionierung und ggf. Heißhaltung der Komponenten entnommen worden. Bei den Erkrankten gelang zwar kein Nachweis des Erregers, Symptome und Verlauf der Erkrankungen ließen jedoch den Schluss zu, dass B. cereus tatsächlich ursächlich für diesen Ausbruch war. Der Infektionsweg bzw. die Kontaminationsquelle konnte jedoch nicht mehr abschließend ermittelt werden.

Ein großer Norovirus-Ausbruch verursachte 73 Erkrankungen in einer Schule mit Gemeinschaftsverpflegung. Ursächliches Lebensmittel waren wahrscheinlich Himbeeren, die mit Panna Cotta serviert worden waren. Der Zusammenhang zwischen dem Verzehr des Lebensmittels und den Erkrankungen konnte durch eine analytische epidemiologische Studie (Kohortenstudie) hergestellt werden.

In einem Landkreis traten ca. zwei Wochen nach Inbetriebnahme eines Rohmilchabgabeautomaten insgesamt 18 Erkrankungen durch Campylobacter jejuni auf. Die Befragung der Betroffenen ergab, dass sie die Rohmilch aus dem Abgabeautomaten ohne vorherige Erhitzung verzehrt hatten, obwohl ein entsprechender Warnhinweis vor Ort angebracht war. Proben der gesammelten Rohmilch aus dem Tank des Erzeugers ergaben zwar keinen Erregernachweis, jedoch konnte in einer Probe aus dem Abgabeautomaten $C$. jejuni nachgewiesen werden. Dies sprach dafür, dass sich durch Mängel bei der Reinigung des Automaten ein Biofilm gebildet hatte, in dem sich der Erreger ansiedeln konnte.

Bei einem kreisübergreifenden Ausbruch in Nordrhein-Westfalen erkrankten insgesamt 26 Kinder zweier Schulklassen. Die Schulklassen hatten auf einem Ferienhof übernachtet und wurden dort auch versorgt. Die Befragungen durch das zuständige Gesundheitsamt ergaben, dass alle Betroffenen Rohmilch bzw. aus Rohmilch hergestellten Kakao getrunken hatten. Die Rohmilch wurde vor der Verwendung nicht abgekocht. Im Rahmen der Ermittlungen stellte sich heraus, dass auch eine Mitarbeiterin des Hofes erkrankt war. In sechs Stuhlproben der Kinder und bei der erkrankten Mitarbeiterin wurde Campylobacter jejuni nachgewiesen.

Zwei kleinere Ausbrüche, die über BELA aus Hamburg und dem Saarland übermittelt worden waren, wurden durch Ciguatoxin in Fisch (Red Snapper) verursacht. Beide Ausbrüche konnten auf dieselbe Charge des Fisches zurückgeführt werden und wurden als ein Ausbruch an die EFSA übermittelt.

\subsection{An die EFSA berichtete lebensmittelbedingte Ausbrüche mit niedriger Evidenz im Jahr 2015}

Daten zu insgesamt 356 lebensmittelbedingten Ausbrüchen mit niedriger Evidenz für den Zusammenhang zwischen einem Lebensmittel und den Erkrankungen wurden aggregiert an die EFSA übermittelt. Auch unter den Ausbrüchen mit niedriger Evidenz waren von Campylobacter verursachte Ausbrüche am häufigsten ( $\mathrm{n}=170,48 \%$ ). Am zweithäufigsten waren Ausbrüche durch Salmonella spp. ( $\mathrm{n}=106,30 \%)$ (Tab. 2). Durch Ausbrüche mit niedriger Evidenz wurden mindestens 1.453 Erkrankungen und 171 Hospitalisierungen verursacht. Bei einigen Ausbrüchen blieb die genaue Zahl der Erkrankungen unbekannt. Bei einem großen Ausbruch mit 217 Erkrankungen, der sich in einer Firmenkantine ereignete, blieb der Erreger unbekannt. Auch das den Ausbruch verursachende Lebensmittel konnte nicht ermittelt werden.

\section{Diskussion}

Daten zu lebensmittelbedingten Ausbrüchen in Deutschland im Jahr 2015, die an die EFSA übermittelt wurden, werden mit diesem Bericht erstmals gemeinsam von BVL und RKI veröffentlicht. In Deutschland werden Daten zu lebensmittelbedingten Ausbrüchen über zwei parallel angelegte Meldesysteme, das IfSG-Meldesystem der Gesundheitsbehörden und das BELA-System der Lebensmittelüberwachungsbehörden, erfasst und über die Behörden des Landes an die des Bundes (BVL und RKI) übermittelt. Nicht für jeden Ausbruch, der über das IfSG-Meldesystem an das RKI übermittelt wird, liegt ein BELA-Meldebogen vor und nicht jeder Ausbruch mit BELA-Meldebogen findet sich im IfSGMeldesystem wieder. Mehrere Gründe kommen dafür in Frage. Den Gesundheitsämtern wird jährlich eine Vielzahl von Krankheitsausbrüchen gemeldet, die in einzelnen Privathaushalten auftraten und jeweils nur wenige Erkrankungsfälle (2 bis 4 Fälle pro Ausbruch) umfassten. Der Verdacht, dass ein kontaminiertes Lebensmittel ursächlich zu den Erkrankungsfällen beigetragen haben könnte, bleibt häufig vage und beruht zum Teil auf reinen Vermutungen der Betroffenen oder des Gesundheitsamtes. Die Mehrzahl dieser Ausbrüche wird hinsichtlich der Aufdeckung der möglichen Infektionsquelle(n) nicht weiter untersucht. Es liegen daher keine spezifischen Informationen $\mathrm{zu}$ einem am Ausbruch beteiligten 
Tab. 2 An die EFSA übermittelte lebensmittelbedingte Ausbrüche mit niedriger Evidenz, nach Erregern, Deutschland, 2015 ( $\mathrm{n}=356$ )

\begin{tabular}{|c|c|c|c|c|c|}
\hline Erreger/Agens & $\begin{array}{l}\text { Anzahl } \\
\text { Ausbrüche }\end{array}$ & $\begin{array}{l}\text { Anteil } \\
\text { (\%) }\end{array}$ & Anzahl Fälle & $\begin{array}{l}\text { Anzahl } \\
\text { Hospitalisierungen }\end{array}$ & $\begin{array}{l}\text { Anzahl } \\
\text { Todesfälle }\end{array}$ \\
\hline Campylobacter spp. & 170 & 47,8 & 463 & 39 & 0 \\
\hline Salmonella Typhimurium & 27 & 7,6 & 99 & 22 & 0 \\
\hline Salmonella Enteritidis & 51 & 14,3 & 164 & 64 & 0 \\
\hline Andere Salmonella Serovare & 28 & 7,9 & 88 & 26 & 0 \\
\hline Norovirus & 20 & 5,6 & 96 & 2 & 0 \\
\hline Parasiten: Giardia & 7 & 2,0 & 17 & 2 & 0 \\
\hline $\begin{array}{l}\text { Enterohämorrhagische } \\
\text { Escherichia coli (EHEC) }\end{array}$ & 6 & 1,7 & 17 & 5 & 0 \\
\hline Hepatitis Virus (A oder E) & 5 & 1,4 & 13 & 5 & 0 \\
\hline Listeria monocytogenes & 3 & 0,8 & 6 & 0 & 0 \\
\hline Shigella & 3 & 0,8 & 6 & 0 & 0 \\
\hline Parasiten: Cryptosporidium & 3 & 0,8 & 6 & 1 & 0 \\
\hline Yersinia enterocolitica & 2 & 0,6 & 4 & 1 & 0 \\
\hline Rotavirus & 2 & 0,6 & 4 & 2 & 0 \\
\hline Clostridium perfringens & 1 & 0,3 & 10 & 0 & 0 \\
\hline Brucella & 1 & 0,3 & 2 & 1 & 0 \\
\hline Bacillus cereus & 1 & 0,3 & Unbekannt & Unbekannt & 0 \\
\hline Unbekannter Erreger & 26 & 7,3 & 458 & 1 & 0 \\
\hline GESAMT & 356 & $100^{a}$ & $1.453^{\mathrm{b}}$ (mind.) & $171^{\mathrm{b}}$ (mind.) & 0 \\
\hline
\end{tabular}

${ }^{a}$ Die berechneten Prozentzahlen wurden gerundet. Deshalb ergibt die Summe nicht genau 100,0\%

${ }^{b}$ Bei einem Ausbruch konnte die Fallzahl bzw. die Anzahl der Hospitalisierungen nicht ermittelt werden. Die Summenangabe stellt deshalb eine Mindestanzahl dar

Lebensmittel vor, die gemäß §27 IfSG an die LMÜ berichtet werden könnten oder die eine sinnvolle BELA-Meldung zum am Ausbruch beteiligten Lebensmittel erlauben würden. Ausbrüche, die durch Erreger oder Agenzien verursacht wurden, die nicht gemäß IfSG meldepflichtig sind (z.B. Bacillus cereus, Clostridium perfringens, Staphylococcus-Enterotoxin, Histamin) oder bei denen der Erreger unbekannt blieb, werden selten an das RKI übermittelt. Vereinzelt finden sich Daten zu derartigen Ausbrüchen in der Übermittlungskategorie „Weitere bedrohliche Gastroenteritiden“. Andere Ausbrüche, für die ein BELA-Bogen vorliegt, sind dem Gesundheitsamt nicht bekannt geworden, z.B. weil die LMÜ von den Erkrankungen über eine Verbraucherbeschwerde Kenntnis erhalten hat, die Betroffenen sich aber nicht in ärztliche Behandlung begeben haben und deshalb keine Meldung gemäß IfSG erfolgte. Zusätzlich wird eine Vielzahl von Ausbrüchen durch meldepflichtige Erreger im IfSG-Meldesystem zwar von den Gesundheitsämtern übermittelt, aber nicht als lebensmittelbedingt deklariert, weil ein möglicher Zusammenhang von epidemiologisch verknüpften Erkrankungen mit einem kontaminierten Lebensmittel nicht vermutet wird oder ein anderer Übertragungsweg, z.B. von Mensch zu Mensch, plausibler erscheint, die Eingaben in die IfSG-Surveillancedatenbank unvollständig sind oder auch weil fälschlicherweise angenommen wird, dass ein Ausbruch nur dann als lebensmittelbedingt übermittelt werden kann, wenn tatsächlich ein Erregernachweis in einem verdächtigten Lebensmittel erfolgte.

Die meisten lebensmittelbedingten Ausbrüche wurden im Jahr 2015 vom Erreger Campylobacter (182/ 384, 47\%) verursacht, darunter waren 12 Ausbrüche mit hoher Evidenz. Bei allen 12 Ausbrüchen war ein Zusammenhang der Erkrankungen mit dem Verzehr von nichterhitzter Rohmilch wahrscheinlich. Bei drei dieser Ausbrüche hatten Schulklassen oder andere Gruppen Rohmilch vom Bauernhof getrunken. Vier Ausbrüche wurden verursacht, weil Personen Rohmilch von Rohmilchabgabeautomaten, sogenannten Milchtankstellen, getrunken hatten, ohne den Hinweis, dass die Rohmilch vor dem Verzehr erhitzt werden sollte, zu beachten. Eine Zunahme von Campylobacter-Ausbrüchen, aber auch von Ausbrüchen z.B. durch enterohämorrhagische $E$. coli (EHEC) oder Salmonellen, durch zunehmenden Konsum von Rohmilch aus Milchtankstellen ist $\mathrm{zu}$ erwarten, weil die Anzahl der Milchtankstellen voraussichtlich weiter zunehmen wird. Mehr und mehr 
Bauern hoffen wegen des Preisrückgangs bei der Milch über den Betrieb von Rohmilchabgabeautomaten auf zusätzliche Einnahmen (Berliner Morgenpost 2016; Handelsblatt 2016). An den Rohmilchabgabestellen müssen Hinweise für die Verbraucher angebracht werden, dass die Rohmilch vor dem Verzehr erhitzt werden sollte. Diese werden von den Verbrauchern aber nicht immer beachtet oder nicht wahrgenommen. Das Bundesinstitut für Risikobewertung (BfR) hat in einer Stellungnahme vom April 2016 noch einmal deutlich auf das gesundheitliche Risiko durch den Verzehr von nichterhitzter Rohmilch hingewiesen (Bundesinstitut für Risikobewertung 2016a, b). Verbrauchern wird geraten, den Hinweis, Rohmilch vor dem Verzehr abzukochen, unbedingt zu befolgen. Auch vom Verzehr von Kakao- oder anderen Milchmixgetränken mit nichterhitzter Rohmilch wird abgeraten (Bundesinstitut für Risikobewertung 2016a, b).

Die Anzahl der Ausbrüche durch Salmonellen und damit deren Anteil an den lebensmittelbedingten Ausbrüchen hat im Vergleich zu den Vorjahren abgenommen. Unter den Ausbrüchen mit hoher Evidenz betrug der Anteil der Salmonellose-Ausbrüche im Jahr 2015 nur noch 11\% (3/28). Seit 2010 (18) 40, 45\%), mit Ausnahme des Jahres 2012, konnte ein kontinuierlicher Rückgang des Anteils der Salmonellose-Ausbrüche an den Ausbrüchen mit hoher Evidenz beobachtet werden. Im Jahr 2014 waren noch 29\% (9/28) der Ausbrüche durch Salmonella verursacht worden. Mit dem rückläufigen Trend von Salmonellosen insgesamt, vor allem der Salmonellosen durch Salmonella Enteritidis, der in den IfSGMeldedaten deutlich ist (Frank et al. 2009), ist eine weitere Abnahme der Salmonellose-Ausbrüche zu erwarten. Eine mögliche Erklärung für den rückläufigen Trend bei den Humanerkrankungen ist der Erfolg des EU-weiten Salmonellen-Bekämpfungsprogramms bei Hühnern gemäß der GeflügelSalmonellen-Verordnung, die z.B. eine Impfpflicht für Legehennen beinhaltet [Verordnung (EG) Nr. 2160/2003; Verordnung zum Schutz gegen bestimmte Salmonelleninfektionen beim Haushuhn und bei Puten 2014].

Im Jahr 2015 wurden 21\% (5/28) der Ausbrüche mit hoher Evidenz durch Histamin in Thunfisch (4 Ausbrüche) oder marines Biotoxin (Cigua-Toxin) in RedSnapper-Fisch verursacht (1 Ausbruch). Ausbrüche mit diesen Agenzien werden der LMÜ meist über Verbraucherbeschwerden bekannt. Gesundheitsämtern liegen zu derartigen Ausbrüchen häufig keine Daten vor, weil die Erkrankten sich nicht in ärztliche Behandlung begeben haben bzw. die
Krankheit nicht meldepflichtig ist. Angaben über die Anzahl der erkrankten Personen können deshalb nicht, wie bei den meisten anderen Ausbrüchen, von den Gesundheitsämtern geliefert werden, sondern beruhen auf Informationen, die der LMÜ vorliegen.

Wie in den Vorjahren wurden auch im Jahr 2015 wieder Hunderte von lebensmittelbedingten Ausbrüchen an die Gesundheits- bzw. Lebensmittelüberwachungsbehörden gemeldet. Über 2.000 Personen sind in Zusammenhang mit diesen Ausbrüchen erkrankt, über 200 davon so schwer, dass sie im Krankenhaus behandelt werden mussten. Es ist davon auszugehen, dass jährlich deutlich mehr lebensmittelbedingte Ausbrüche und Erkrankungen auftreten, die gemeldeten Ausbrüche also nur die Spitze des Eisbergs darstellen. Ausbrüche werden den Behörden nicht bekannt, wenn die Betroffenen sich nicht in ärztliche Behandlung begeben, bei den Erkrankten kein Erregernachweis angestrebt wird oder die Krankheiten nicht meldepflichtig sind. Möglich ist auch, dass ein Ausbruchsgeschehen nicht als solches erkannt wird, weil ein Zusammenhang zwischen den Erkrankungen nicht offensichtlich ist, oder ein Ausbruchsgeschehen zwar erkannt, aber nicht als lebensmittelbedingt eingeschätzt wird. So wurden beispielsweise im Jahr 2015 von 465 nach IfSG übermittelten Krankheitsausbrüchen, die von Campylobacter spp. verursacht wurden, lediglich $38 \%$ als lebensmittelbedingt übermittelt, obwohl davon auszugehen ist, dass die meisten dieser Ausbrüche über ein kontaminiertes Lebensmittel verursacht wurden.

Gemeinsame, interdisziplinäre Ausbruchsuntersuchungen durch die zuständigen Gesundheitsämter und Lebensmittelüberwachungsbehörden sind unerlässlich, um die Ausbruchsursachen zu erkennen und geeignete Maßnahmen ergreifen sowie Empfehlungen aussprechen zu können, die den aktuellen Ausbruch stoppen und weitere Ausbrüche verhindern können. Nur durch die Kombination der verschiedenen Kompetenzen und Zuständigkeiten wird eine lückenlose Aufklärung des Ausbruchsgeschehens möglich. Insbesondere in Krisensituationen ist es von Vorteil, wenn die Akteure sich bereits kennen und gemeinsame Vorgehensweisen eingespielt sind.

Für Mitarbeiterinnen und Mitarbeiter im Öffentlichen Gesundheitsdienst werden zum Thema Ausbruchsuntersuchungen verschiedene Weiterbildungsmöglichkeiten angeboten. Leitfäden für die Untersuchung von lebensmittelbedingten Infektionen und Ausbrüchen werden z.B. vom BfR und von den Landesgesundheits- und -veterinärbehörden in 
Baden-Württemberg bereitgestellt (Bundesinstitut für Risikobewertung 2014; Landesgesundheitsamt Baden-Württemberg und Chemisches und Veterinäruntersuchungsamt Stuttgart 2015). Eine jährliche Schulung zur Vorgehensweise bei Ausbruchsuntersuchungen bietet das RKI für Mitarbeiterinnen und Mitarbeiter in Gesundheitsämtern und Landesgesundheitsbehörden (Epikurs@RKI, Modul „Ausbrüche untersuchen“) an. Wünschenswert wäre es, wenn auch lokale Ausbrüche häufiger mit analytischen epidemiologischen Studien untersucht werden würden, sofern diese sinnvoll erscheinen. Bei vielen lebensmittelbedingten Ausbrüchen gelingt ein mikrobiologischer Nachweis des Erregers im verdächtigten Lebensmittel nicht oder es sind keine Lebensmittelproben mehr vorhanden, die untersucht werden könnten. Analytische epidemiologische Studien, bei denen sowohl Erkrankte als auch Nicht-Erkrankte hinsichtlich ihres Lebensmittelverzehrs befragt und verglichen werden, können auf das Lebensmittel, das den Ausbruch verursacht haben könnte, hinweisen und so die Grundlage für Maßnahmen oder weiterführende Untersuchungen, z.B. Lebensmittelrückverfolgungen, darstellen. Im Jahr 2015 wurde dem BVL bzw. dem RKI nur ein einziger Ausbruch übermittelt, bei dem die Evidenz für den Zusammenhang zwischen Lebensmittel und Erkrankungen über eine analytische Studie (Kohortenstudie) erlangt wurde. Auch in den Vorjahren wurden meist nur 1-2 lebensmittelbedingte Ausbrüche pro Jahr mit Hilfe einer analytischen epidemiologischen Studie untersucht. Meist waren dies überregionale Ausbrüche oder Ausbrüche, die mehrere Bundesländer betrafen. Hier sind Anstrengungen im öffentlichen Gesundheitsdienst nötig, um die Anzahl der analytischepidemiologischen Untersuchungen bei lokalen und überregionalen (mutmaßlich) lebensmittelbedingten Ausbrüchen zu erhöhen. Zur Unterstützung von Untersuchungen lokaler lebensmittelbedingter Ausbrüche mit analytischen epidemiologischen Studien bietet das RKI ein Excel-basiertes Analysewerkzeug („,Linelist-Werkzeug“) an, das über die RKI-Webseite erhältlich ist (Werber and Bernard 2014). ${ }^{1}$ Die Anwendungsmöglichkeiten dieses Analysetools werden im oben genannten Kursmodul des RKI vermittelt. Die in der Lebensmittelüberwachung tätigen Mitarbeiterinnen und Mitarbeiter können sich regelmäßig in Veranstaltungen, die von den Landesbehörden bzw. den Berufsverbänden angeboten werden, fortbilden. Hierbei werden auch das

$\overline{1}$ http://www.rki.de/linelisttool.
Vorgehen bei lebensmittelbedingten Ausbrüchen und die Zusammenarbeit mit den Gesundheitsämtern thematisiert. Die Europäische Kommission hat ein eigenes Trainingsprogramm initiiert (,Better Training for Safer Food“), das spezielle Trainingskurse zur Aufklärung lebensmittelbedingter Krankheitsausbrüche beinhaltet. ${ }^{2}$

Auch durch die Aufklärung von Verbraucherinnen und Verbrauchern über die gesundheitlichen Risiken, die mit dem Verzehr bzw. der Zubereitung bestimmter Lebensmittel einhergehen können, können lebensmittelbedingte Ausbrüche vermieden werden. Aufklärungsmaterial zu einer Vielzahl von lebensmittelbedingten Infektionen und Ergebnisse von Risikobewertungen werden auf der Webseite des BfR bereitgestellt und können hier nur beispielhaft erwähnt werden (Bundesinstitut für Risikobewertung 2015b, c). Auch regelmäßige Schulungen von Personal in Gaststätten und Gemeinschaftseinrichtungen über den richtigen Umgang mit Lebensmitteln können zur Vermeidung von Ausbrüchen beitragen (Bundesinstitut für Risikobewertung 2015a, d, 2016c).

Open Access This article is distributed under the terms of the Creative Commons Attribution 4.0 International License (http://creativecommons.org/licenses/by/4.0/), which permits unrestricted use, distribution, and reproduction in any medium, provided you give appropriate credit to the original author(s) and the source, provide a link to the Creative Commons license, and indicate if changes were made.

\section{Literatur}

AVV Zoonosen Lebensmittelkette (2012) Allgemeine Verwaltungsvorschrift über die Erfassung, Auswertung und Veröffentlichung von Daten über das Auftreten von Zoonosen und Zoonoseerregern entlang der Lebensmittelkette. BAnz 2012, Nr. 27 S. 623, Geändert durch Verwaltungsvorschrift vom 20. Oktober 2014 (BAnz AT 07.11.2014 B2)

Berliner Morgenpost (2016) Warum Bauern immer häufiger auf Milchtankstellen setzen. http://www.morgenpost.de/ wirtschaft/article208065637/Warum-Bauern-immer-haeufiger-auf-Milchtankstellen-setzen.html. Zugriff am 20.09.2016

Bundesinstitut für Risikobewertung (2014) Ausbruchsaufklärung entlang der Lebensmittelkette. Leitfaden einer am BfR eingerichteten Bund-Länder-Arbeitsgruppe zur Erarbeitung eines Leitfadens zur Ausbruchsaufklärung entlang der Lebensmittelkette http://www.bfr.bund.de/cm/343/ausbruchsaufklaerung-entlang-der-lebensmittelkette.pdf. Zugriff am 16. November 2016

Bundesinstitut für Risikobewertung (2015a) An Krankheitsausbrüchen beteiligte Lebensmittel in Deutschland im

\footnotetext{
2 http://www.trainsaferfood.eu/Trainings/

Foodborneoutbreaksinvestigation.aspx.
} 
Jahr 2014. http://www.bfr.bund.de/cm/343/an-krankheitsausbruechen-beteiligte-lebensmittel-in-deutschland-im-jahr2014.pdf. Zugriff am 16. November 2016

Bundesinstitut für Risikobewertung (2015b) Merkblätter für Verbraucher: Schutz vor lebensmittelbedingten Infektionen mit Campylobacter. http://www.bfr.bund.de/cm/350/ verbrauchertipps-schutz-vor-lebensmittelbedingten-infektionen-mit-campylobacter.pdf. Zugriff am 16. November 2016

Bundesinstitut für Risikobewertung (2015c) Merkblätter für Verbraucher: Schutz vor Lebensmittelinfektionen im Privathaushalt. http://www.bfr.bund.de/cm/350/verbrauchertipps_schutz_vor_lebensmittelinfektionen_im_privathaushalt.pdf. Zugriff am 16. November 2016

Bundesinstitut für Risikobewertung (2015d) Sicher verpflegt. Besonders empfindliche Personengruppen in Gemeinschaftseinrichtungen. http://www.bfr.bund.de/cm/350/ sicher-verpflegt-besonders-empfindliche-personengruppenin-gemeinschaftseinrichtungen.pdf. Zugriff am 16. November 2016

Bundesinstitut für Risikobewertung (2016a) Rohmilch: Abkochen schützt vor Infektion mit Campylobacter. Stellungnahme Nr. 008/2016 des BfR vom 13. April

Bundesinstitut für Risikobewertung (2016b) FAQ zum Verzehr von Rohmilch http://www.bfr.bund.de/cm/343/fragen-undantworten-zum-verzehr-von-rohmilch.pdf. Zugriff am 15 November 2016

Bundesinstitut für Risikobewertung (2016c) Hygieneregeln in der Gemeinschaftsgastronomie. http://www.bfr.bund.de/ $\mathrm{cm} / 350 /$ hygieneregeln-in-der-gemeinschaftsgastronomiedeutsch.pdf. Zugriff am 15 November 2016

European Food Safety Authority (2011) Updated technical specifications for harmonised reporting of foodborne outbreaks through the European Union reporting system in accordance with Directive 2003/99/EC. EFSA J 9(4):2101

European Food Safety Authority (2016) Manual for reporting on food-borne outbreaks in accordance with Directive 2003/99/EC for information deriving from the year 2015. EFSA supporting publication. doi:10.2903/sp.efsa.2016.EN989
Frank C, Käsbohrer A, Stark K, Werber D (2009) Marked decrease in reporting incidence of salmonellosis driven by lower rates of Salmonella Enteritidis infections in Germany in 2008: a continuing trend. Euro Surveill 14(11):1-2

Handelsblatt (2016) Bauern setzen auf eigene Tankstellen. http://www.handelsblatt.com/unternehmen/handel-konsumgueter/milchpreis-bauern-setzen-auf-eigene-tankstellen/ 14012844.html. Zugriff am 25.10.2016

Landesgesundheitsamt Baden-Württemberg und Chemisches und Veterinäruntersuchungsamt Stuttgart (2015) Leitfaden Management lebensmittelassoziierter Infektionen in Baden-Württemberg. Handlungsempfehlungen einer interdisziplinären Arbeitsgruppe. http://www.cvuas.de/ pub/beitrag.asp?ID=2177\&subid=0\&Thema_ID $=2 \& P d f=$ Yes. Zugriff am 16. November 2016

Richtlinie 2003/99/EG des Europäischen Parlaments und des Rates vom 17. November 2003 zur Überwachung von Zoonosen und Zoonoseerregern und zur Änderung der Entscheidung 90/424/EWG des Rates sowie zur Aufhebung der Richtlinie 92/117/EWG des Rates, ABl. L 325, 12.12.2003

Robert Koch-Institut (2016) Infektionsepidemiologisches Jahrbuch für 2015. http://www.rki.de/DE/Content/Infekt/Jahrbuch/Jahrbuch_2015.pdf?_blob=publicationFile. Zugriff am 16.November 2016

Verordnung (EG) Nr. 2160/2003 des Europäischen Parlaments und des Rates vom 17. November 2003 zur Bekämpfung von Salmonellen und bestimmten anderen durch Lebensmittel übertragbaren Zoonoseerregern

Verordnung zum Schutz gegen bestimmte Salmonelleninfektionen beim Haushuhn und bei Puten (2014) GeflügelSalmonellen-Verordnung in der Fassung der Bekanntmachung vom 17. Januar (BGBl. I S. 58), die zuletzt durch Artikel 9 der Verordnung vom 29. Dezember 2014 (BGBl. I S. 2481) geändert worden ist

Werber D, Bernard H (2014) Reducing the barriers against analytical epidemiological studies in investigations of local foodborne disease outbreaks in Germany-a starter kit for local health authorities. Euro Surveill 19(8):20714 